PREHOSPITAL CARE

\title{
Potential impact of interventions to reduce times to thrombolysis
}

\author{
S Goodacre, A-M Kelly, D Kerr
}

Emerg Med J 2004;21:625-629. doi: 10.1136/emj.2003.012575

See end of article for authors' affiliations

......................

Correspondence to:

Dr S W Goodacre,

Medical Care Research

Unit, Regent Court,

30 Regent Street, Sheffield

S1 4DA, UK; s.goodacre@

sheffield.ac.uk

Accepted for publication 13 May 2004
Objectives: To estimate the lifesaving potential of interventions to accelerate the administration of intravenous thrombolysis for myocardial infarction.

Methods: Data were analysed from a prospective, observational study of all patients transported to hospital by ambulance, who subsequently received intravenous thrombolysis at 20 hospitals and two ambulance services in Victoria, Australia $(n=1147)$. Regression models estimated the association between predictor variables age, sex, route of referral, symptom onset to call time, ambulance pre-notification of the receiving hospital, emergency department thrombolysis, and the outcome, time to thrombolysis. Further modelling estimated the number needed to treat to save one life by several recommended interventions to reduce time delays.

Results: Presentation via a rural hospital or general practitioner was associated with an approximate doubling of the onset to call time (2.08 and 2.30 respectively). Ambulance-hospital pre-notification and emergency department thrombolysis reduced door to needle times by $21 \%$ and $27 \%$ respectively. Modelling showed that each of the following interventions would be expected to save one life: 1069 hospital pre-notifications, 714 cases of emergency department thrombolysis, 184 cases of prehospital thrombolysis, 340 cases to bypass their rural hospital, or 50 cases to bypass their general practitioner. Conclusions: Hospital pre-notification and emergency department thrombolysis reduce time delays, although the mortality impact seems to be modest. Prehospital thrombolysis has the potential to save lives, although validation in real practice is required. Advising patients to call directly for an ambulance, rather than the general practitioner, has the greatest potential to save lives. e ntravenous thrombolytic therapy is established as an effective treatment for acute myocardial infarction (AMI). There is clear evidence that the effectiveness of thrombolysis is related to the time delay between onset of symptoms and administration of therapy. ${ }^{1}$ This relation has been modelled and appears to be non-linear. ${ }^{2}$ Treatment effect declines as time delay increases, but with a diminishing rate of decline over time. The practical implication of this is that the effect upon mortality of interventions that reduce times to thrombolysis will be dependent upon the overall time delay. For example, saving 10 minutes will be much more valuable if the overall time to thrombolysis is one hour, than if it were four hours. Boersma et al estimated that the relation between effect and time delay was best described by the equation:

Mortality reduction per treated 1000 patients $=$ $19.4-0.6 \mathrm{x}+29.3 \mathrm{x}^{-1}$

(where $\mathrm{x}$ is the time delay, in hours, between symptom onset and treatment).

A recent study of call to needle (CTN) times for thrombolysis in ST elevation AMI in Victoria, Australia established that many patients had significant delays before receiving thrombolysis and may have incurred potentially avoidable mortality. ${ }^{3}$ A number of interventions have been suggested to reduce such delays. These include ambulance pre-notification of receiving hospitals, ${ }^{4}$ thrombolysis in the emergency department (ED) rather than the coronary care unit (CCU), ${ }^{5}$ prehospital thrombolysis, ${ }^{6}$ bypass of rural hospitals if they are unable to deliver thrombolysis, and advising patients to call directly for an ambulance rather than their GP if they experience chest pain. $^{7}$
These interventions all, to varying degrees, require reorganisation of services. Any change in service delivery will cause disruption and consume scarce resources, and risks making care worse, rather than better. It is therefore essential that before any change is made we estimate the potential benefit of intervention, so that we can then judge whether this justifies the potential costs. The Boersma equation fulfils a crucial role in this respect, in that it allows us to convert a measure of benefit that is somewhat meaningless to the patient or health service (time saved) to one that is meaningful and important (lives saved). If we know how many lives should be saved by an intervention we can relate this to the potential costs to estimate whether the intervention is likely to be considered worthwhile.

We aimed to estimate the potential impact, in terms of additional lives saved, of each of the above interventions, by modelling data from the Victorian CTN study ${ }^{3}$ with the Boersma estimate of the relation between mortality and treatment effect. ${ }^{2}$

\section{METHODS}

The methodology of the Victorian CTN study has been reported in detail elsewhere. ${ }^{3}$ Observational data were collected from 20 hospitals and two ambulance services over a 30 month period for all patients arriving at hospital by ambulance who received thrombolysis. The data collected represent the vast majority of eligible patients in Victoria. Ethics committee approval for the study was provided by all the participating hospitals.

Abbreviations: $E D$, emergency department; $A M I$, acute myocardial infarction; CTN, call to needle; CCU, coronary care unit; OTC, onset to call; CTD, call to dispatch; DTS, dispatch to scene; DTN, door to needle 
Data from this study were entered into linear regression models to predict symptom onset to call (OTC) times, call to dispatch (CTD) times, dispatch to scene (DTS) times, on scene times, transport times, and door to needle (DTN) times. Because these outcomes are positively skewed, all time to event data were log-transformed. Age, sex, and route of referral (direct, via GP, or via rural hospital) were used to predict OTC times. Age, sex, route of referral, and OTC time were used to predict CTD, DTS, on scene time, and transport time. Age, sex, route of referral, OTC time, ambulance prenotification of hospital, and emergency department (ED) thrombolysis were used to predict DTN and CTN times. Coefficients from these regression models were back transformed to give an estimate of the relative effect of each covariate upon each relevant time period.

For each patient in the Victorian study an estimate of their predicted probability of mortality was generated by applying the Boersma equation to their total time from symptom onset to thrombolysis. These values were then summed and divided by the total cohort to give the overall probability of mortality for the cohort.

The effect of each of the potential interventions upon mortality was modelled as follows:

\section{Ambulance pre-notification}

All patients in the cohort for whom the ambulance service did not provide pre-notification to the receiving hospital were selected. Their DTN times were multiplied by the estimated effect of pre-notification and their expected mortality was recalculated. The overall change in mortality for these patients was estimated by summing the change in mortality for each patient and dividing by their total number.

\section{ED thrombolysis}

All patients in the cohort who received thrombolysis in a CCU were selected. Their DTN times were multiplied by the estimated effect of ED thrombolysis and their expected mortality was recalculated. The overall change in mortality for these patients was estimated by summing the change in mortality for each patient and dividing by their total number.

\section{Prehospital thrombolysis}

To estimate the potential impact of prehospital thrombolysis we first assumed that all patients could receive prehospital thrombolysis and that this would effectively remove the on scene and transport times. We also assumed that paramedics would provide thrombolysis with the same efficiency as the ED. So DTN times were used to estimate the time from ambulance arrival to thrombolysis administration. As before, the change in mortality was estimated using the Boersma equation.

Recognising that patients with greater travel distance to a treating hospital are more likely to have longer CTN times and thus may have greater benefit from prehospital thrombolysis, we also modelled the impact of prehospital thrombolysis for patients with transport times to hospital of greater than 30 minutes. The same assumptions regarding times were made as for the whole cohort and the estimated change in mortality calculated the same way.

\section{Rural hospital bypass}

To model the effect of bypassing rural hospitals, we used the estimated effect of being referred via a rural hospital generated by regression modelling. The OTC times of all patients referred via a rural hospital were divided by the estimated effect of referral by a rural hospital. Mortality change was then modelled using the Boersma equation.

\section{Call for ambulance rather than GP}

To model the effect of calling an ambulance directly rather than their GP, we used the estimated effect of referral via a GP generated by regression modelling. The OTC times of all patients referred via their GP were divided by the estimated effect of referral via GP. Mortality change was then modelled using the Boersma equation.

Linear regression modelling was undertaken using SPSS for Windows (version 10, SPSS, Chicago, IL). Modelling of time delay and mortality using the Boersma equation was undertaken using a Microsoft Excel spreadsheet.

\section{RESULTS}

The cohort consisted of 1147 patients with a mean age of 65.7 years (median 67 years, range 21 to 93 years). There were 350 women $(30.5 \%)$ and 797 men $(69.5 \%)$. The route of referral was by direct call to ambulance in 772 cases $(67.3 \%)$, via GP referral in 180 cases $(15.7 \%)$, via a rural hospital in 50 cases $(4.4 \%)$, and by other means in 141 cases $(12.3 \%)$. ED thrombolysis was provided for 896 (78.1\%), while 192 $(16.7 \%)$ received thrombolysis on CCU and $59(5.1 \%)$ received thrombolysis elsewhere. Ambulance pre-notification of the receiving hospital occurred for 482 patients (42.0\%).

The mean (median) time delays for the cohort were: OTC 131 (57) minutes, CTD 3 (2) minutes, DTS 11 (9) minutes, scene time 16 (15) minutes, transport time 17 (15) minutes, DTN 47 (37) minutes, and CTN 93 (83) minutes. The observed mortality for the cohort was 98 of 1147 patients, or $8.5 \%$ (95\% CI 7.1 to 10.3$)$.

Tables 1 to 6 outline the results of regression modelling. For continuous variables (age and OTC time) the values reported estimate the relative effect upon the time to event of each unit change in predictor variable (that is, for each year of life or each minute of OTC time). For categorical variables the values reported estimate the relative effect upon time to event of that variable being positive.

Older patients had longer OTC and DTN times. OTC times were increased by $0.9 \%$ for every year of age, while DTN times increased by $0.6 \%$ for every year of age. Sex did not predict any outcome.

All times, except DTN times, were prolonged for patients initially attending rural hospitals. In general, times for these patients were doubled, compared with patients who called for an ambulance that took them directly to hospital. Patients who were referred via their GP had increased OTC and CTD

\begin{tabular}{llll}
\hline \multicolumn{3}{l}{ Table 1} & Prediction of onset to call time \\
\hline Factor & Relative effect & $95 \% \mathrm{Cl}$ & p Value \\
\hline Age & 1.009 & 1.003 to 1.016 & 0.004 \\
Male sex & 0.91 & 0.77 to 1.08 & 0.268 \\
Source: GP & 2.30 & 1.86 to 2.83 & $<0.001$ \\
Source: Other & 0.59 & 0.46 to 0.74 & $<0.001$ \\
Source: Rural & 2.08 & 1.44 to 3.01 & $<0.001$ \\
Source: Home & Reference & - & - \\
\hline
\end{tabular}




\begin{tabular}{|c|c|c|c|}
\hline Factor & Relative effect & $95 \% \mathrm{Cl}$ & p Value \\
\hline Age & 0.999 & 0.995 to 1.003 & 0.714 \\
\hline Male sex & 0.96 & 0.87 to 1.07 & 0.500 \\
\hline Onset to call time & 1.000 & 0.999 to 1.000 & 0.799 \\
\hline Source: GP & 1.17 & 1.03 to 1.34 & 0.017 \\
\hline Source: Other & 1.19 & 1.03 to 1.37 & 0.016 \\
\hline Source: Rural & 2.94 & 2.32 to 3.73 & $<0.001$ \\
\hline Source: Home & Reference & - & - \\
\hline
\end{tabular}

Table 3 Prediction of dispatch to scene time

\begin{tabular}{llll}
\hline Factor & Relative effect & $\mathbf{9 5 \%} \mathbf{C l}$ & $\mathbf{p ~ V a l u e}$ \\
\hline Age & 0.998 & 0.995 to 1.000 & 0.140 \\
Sex & 1.03 & 0.95 to 1.12 & 0.500 \\
Onset to call time & 1.000 & 0.999 to 1.000 & 0.190 \\
Source: GP & 0.88 & 0.79 to 0.98 & 0.019 \\
Source: Other & 0.87 & 0.77 to 0.98 & 0.019 \\
Source: Rural & 2.00 & 1.66 to 2.42 & $<0.001$ \\
Source: Home & Reference & - & - \\
\hline
\end{tabular}

times, but shorter dispatch and scene times. Transport times and DTN times were unaffected.

Ambulance pre-notification and ED thrombolysis both reduced DTN times, with relative reductions of $21 \%$ and $27 \%$ respectively. OTC time predicted DTN time, although with each hour of prolonged OTC time predicting a $1 \%$ relative increase in DTN time, this was unlikely to be clinically significant.

Ambulance pre-notification of receiving hospitals was performed for $42 \%$ of the study cohort. Modelling the effect of ambulance pre-notification in the remaining 58\% produced an estimated $0.09 \%$ absolute reduction in their expected mortality. Pre-notification of 1069 cases would thus be required to save one life.

Only $17 \%$ of the cohort were transferred to CCU for thrombolysis. If these patients had been thrombolysed in the ED their expected mortality could have been reduced by $0.14 \%$. Hence 714 additional patients would have to receive ED thrombolysis to save one life.

Introducing prehospital thrombolysis for the whole cohort produced an estimated $0.54 \%$ absolute reduction in mortality. Hence, under the assumptions of the model, we would need to provide prehospital thrombolysis for 184 patients to save one life. For the subgroup with transport times of longer than 30 minutes, it is estimated that prehospital thrombolysis would produce an estimated $0.6 \%$ reduction in mortality, or require 168 patients to receive prehospital thrombolysis to save one life. Using the second approach only 124 patients in the cohort would receive prehospital thrombolysis.
Bypassing rural hospitals was estimated to reduce mortality by $0.29 \%$, so 340 patients would need to be managed in this way to save one life. However, only 50 patients in the study cohort would have been eligible. Calling directly for an ambulance rather than the GP was estimated to reduce mortality by $0.5 \%$, so we might expect one life to be saved for every 200 patients who called directly for an ambulance rather than their GP.

\section{DISCUSSION}

The Victorian call to needle time study identified delays in the administration of thrombolysis and highlighted potential interventions to reduce these delays. ${ }^{3}$ Simply knowing that an intervention might reduce time delay is not sufficient to justify the potential costs of implementation. Before introducing any of these interventions we need to know whether the potential benefit is likely to justify the costs. In this study we have modelled the original study data using the Boersma equation to estimate the potential benefit, in terms of lives saved, of five measures to improve thrombolysis times. It has shown modest reductions in estimated mortality for the interventions of pre-notification of hospitals, ED thrombolysis, and bypassing rural hospitals and moderate reductions in estimated mortality for prehospital thrombolysis and calling directly for an ambulance.

Modelling is often used to inform health service policy and derive estimates of the potential health impact of policy changes. Yet it is often unclear how estimates are derived, and whether they are likely to be valid or applicable to a particular population. These data, derived from observation

Table 4 Prediction of scene time

\begin{tabular}{llll}
\hline Factor & Relative effect & $95 \% \mathrm{Cl}$ & $\mathrm{p}$ Value \\
\hline Age & 0.999 & 0.996 to 1.001 & 0.240 \\
Sex & 0.95 & 0.90 to 1.02 & 0.145 \\
OTC & 1.000 & 0.999 to 1.000 & 0.340 \\
Source: GP & 0.81 & 0.75 to 0.88 & $<0.001$ \\
Source: Other & 0.86 & 0.79 to 0.94 & 0.001 \\
Source: Rural & 1.17 & 1.02 to 1.35 & 0.023 \\
Source: Home & Reference & - & - \\
\hline
\end{tabular}




\begin{tabular}{|llll|}
\hline \multicolumn{3}{l}{ Table 5} & Prediction of transport time \\
\hline Factor & Relative effect & $95 \% \mathrm{Cl}$ & p Value \\
\hline Age & 0.999 & 0.996 to 1.002 & 0.338 \\
Sex & 1.01 & 0.93 to 1.09 & 0.809 \\
Onset to call & 1.000 & 0.999 to 1.000 & 0.762 \\
Source: GP & 0.98 & 0.89 to 1.09 & 0.731 \\
Source: Other & 0.90 & 0.80 to 1.00 & 0.058 \\
Source: Rural & 2.50 & 2.09 to 2.98 & $<0.001$ \\
Source: Home & Reference & - & - \\
\hline
\end{tabular}

data in a large cohort, may provide a more reliable reflection of the real world.

Before we try to interpret these results we must consider the limitations of modelling and the potential costs of implementing the findings. Modelling invariably involves assumptions that may or may not be justified. In many cases these assumptions will lead to an overestimate of the potential impact of intervention. For example, estimates of the effect of pre-notification, ED thrombolysis, or route of referral derived from observational data may be subject to confounding. Also, modelling the impact of interventions upon mortality assumes that these interventions can be effectively and efficiently introduced. For example, we assumed that prehospital thrombolysis would result in paramedics providing thrombolysis on scene with the same degree of efficiency as the ED. This discounts the potential difficulties of providing prehospital thrombolysis.

The potential value of each intervention cannot be determined simply from its potential impact upon mortality. We must also consider the cost of implementation, the practicality of implementation, the number of cases potentially eligible for the intervention, and the knock on effects on other patients within the emergency health care system. For example, implementation of prehospital thrombolysis by paramedics would consume a greater period of time than current stabilisation and transfer. Unless numbers of paramedic crews were increased commensurately, access to advanced skills ambulance crews would be reduced for other patients who might benefit from their care.

Taking these issues into account it is probably reasonable to conclude that ambulance pre-notification and ED thrombolysis are likely to have a small, but worthwhile, impact upon mortality. Evidence from other settings supports these findings. ${ }^{45-11}$ Both are practical and already widely used, and are unlikely to incur substantial costs if implemented more extensively. Thus it is reasonable to recommend that, in the absence of specific barriers to their feasibility, these measures should be standard practice. However, it would be wise to estimate the potential effect of over-triage. For example, if only one out of every two cases pre-notified by the ambulance service are actually eligible for thrombolysis, then the number of patients requiring pre-notification to save one life will be doubled.

In the United Kingdom, both the National Service Framework for Coronary Heart Disease, ${ }^{7}$ and the national telephone advice line, NHS Direct, ${ }^{12}$ advise patients to call for an ambulance rather than their GP if they have symptoms suggestive of AMI. This study suggests that such advice is well founded and could have a worthwhile effect upon mortality. However, it is based upon the assumption that the delay is entirely attributable to referral via the GP. If these patients wait longer than directly referred patients before calling their GP then the effect upon mortality is likely to be overestimated.

An unintended consequence of such an approach could be increased numbers of patients using the ambulance service and ED with suggestive symptoms but without AMI. It is therefore important that both services develop systems to manage this contingency. As with ambulance pre-notification, an estimate of the potential effect of over-triage would also be valuable.

Bypassing rural hospitals produced a rather smaller effect than would be expected from the substantial time saving that we estimated would be gained by this change (an estimated halving of OTC times). This is because subsequent transport times mean that, even if their OTC times are reduced, the total time to thrombolysis remained long. Because the Boersma curve is non-linear, time changes for patients with shorter overall times have a more dramatic effect on mortality than those in patients with longer overall times.

This suggests that providing thrombolysis at rural hospitals might be more effective than bypassing rural hospitals, although we cannot model this intervention from our data. Whichever approach is used, any intervention will not have a dramatic effect on overall mortality because only 50 patients in this cohort were referred via a rural hospital.

The analysis suggested that prehospital thrombolysis might have a valuable impact in reducing avoidable mortality. This conclusion needs to be viewed with caution. The modelling involved a number of assumptions relating to the efficiency with which prehospital thrombolysis could be administered that have yet to be substantiated. Unlike pre-notification and ED thrombolysis, the estimated effect of prehospital

Table 6 Prediction of door to needle time

\begin{tabular}{llll}
\hline Factor & Relative effect & $\mathbf{9 5 \%} \mathrm{Cl}$ & $\mathbf{p}$ Value \\
\hline Age & 1.006 & 1.003 to 1.009 & $<0.001$ \\
Sex & 0.95 & 0.87 to 1.03 & 0.198 \\
Onset to call time & 1.00015 & 1.0001 to 1.0002 & 0.006 \\
Pre-notification & 0.79 & 0.73 to 0.85 & $<0.001$ \\
ED thrombolysis & 0.73 & 0.67 to 0.80 & $<0.001$ \\
Source: GP & 0.96 & 0.87 to 1.06 & 0.441 \\
Source: Other & 0.93 & 0.84 to 1.05 & 0.238 \\
Source: Rural & 0.87 & 0.73 to 1.04 & 0.132 \\
Source: Home & Reference & - & -
\end{tabular}


thrombolysis was based purely upon assumed time savings, rather than empirical (albeit observational) data.

Introducing prehospital thrombolysis would require substantial reorganisation and incur substantial costs. Our, perhaps over-optimistic, assumption was that 184 patients would need to receive prehospital thrombolysis to save one life. By drawing on experience elsewhere to estimate the potential number of patients receiving prehospital thrombolysis ${ }^{13-16}$ and by estimating the potential cost of establishing prehospital thrombolysis, ${ }^{17}$ this figure could be used to estimate the potential cost effectiveness of introducing prehospital thrombolysis.

Restriction of prehospital thrombolysis to only those patients with long anticipated transport times might seem to be a rational way of increasing the effectiveness of prehospital thrombolysis. Our data suggest that the effect of this approach is attenuated by patients with transport times over 30 minutes also having longer OTC, CTD, and DTS times (means of 183, 5, and 19 minutes respectively). Hence, these patients have already missed much of the potential benefit from thrombolysis by the time an ambulance arrives to treat them.

For an intervention to have a worthwhile effect upon mortality it needs to be applicable to a sufficient number of patients. Rural hospital bypass was only applicable to 50 patients and prehospital thrombolysis for those with long transport times was only applicable to 124 patients. This creates a particular problem for rural patients. In terms of cost effectiveness it is likely that any intervention aimed at rural patients will appear less attractive than one aimed at the more numerous urban population. We are therefore faced with a conflict between efficiency (maximising overall health gain for the population) and equity (ensuring that everyone has an equal opportunity to benefit from health care).

\section{CONCLUSION}

ED thrombolysis and ambulance pre-notification of receiving hospitals seem to have a small but worthwhile effect upon predicted mortality and could be considered standard practice. Advising patients to call directly for an ambulance, rather than their GP, in the presence of symptoms suggestive of AMI can produce a more substantial effect.

Patients attending rural hospitals present a particular problem. Because of their small numbers, interventions to reduce thrombolysis times are unlikely to be considered an efficient use of health service resources, but considerations of equity mean that these patients should not be neglected.

Prehospital thrombolysis offers the potential to reduce mortality but at substantial cost. Further empirical validation of the time savings and cost effectiveness of this approach is required before it can be recommended.

\section{Authors' affiliations}

S Goodacre, Health Service Research and Emergency Medicine, Medical Care Research Unit, University of Sheffield, UK

A-M Kelly, D Kerr, Joseph Epstein Centre for Emergency Medicine Research, Melbourne, Australia

Funding: The Victorian call-to-needle time study was supported by a grant from the Australian Rotary Health Research Fund. Steve Goodacre was supported by a NHS R\&D Health Services Research Fellowship.

Conflicts of interest: none declared.

\section{REFERENCES}

1 Fibrinolytic Therapy Trialists' (FTT) Collaborative Group. Indications for fibrinolytic therapy in suspected acute myocardial infarction: collaborative overview of early mortality and major morbidity results from all randomised controlled trials of more than 1000 patients. Lancet 1994:343:311-22.

2 Boersma E, Maas AC, Deckers JW, et al. Early thrombolytic treatment in acute myocardial infarction: reappraisal of the golden hour. Lancet 1996;348:771-5.

3 Kelly A-M, Kerr D, Patrick I, et al. Call to needle times for thrombolysis in acute myocardial infarction in Victoria. Med J Aust 2003;178:381-5.

4 Millar-Craig MW, Joy AV, Adamowicz M, et al. Reduction in treatment delay by paramedic ECG diagnosis of myocardial infarction with direct CCU admission. Heart 1997;78:456-61.

5 Hourigan CT, Mountain D, Langton PE, et al. Changing the site of delivery of thrombolytic treatment for acute myocardial infarction from the coronary care unit to the emergency department greatly reduces door to needle time. Heart 2000;84:573-4.

6 Morrison LJ, Verbeek PR, McDonald AC, et al. Mortality and prehospital thrombolysis for acute myocardial infarction: a meta-analysis. JAMA 2000;238:2686-92

7 Department of Health. The National Service Framework for coronary heart disease. London: Department of Health, 2000

8 Baneriee S, Rhoden WE. Fast-tracking of myocardial infarction by paramedics. J R Coll Physicians Lond 1998:32:36-8.

9 Bryant M, Kelly AM. "Point of entry" treatment gives best time to thrombolysis for acute myocardial infarction. Aust Health Rev 2001;24:157-60.

10 Edhouse JA, Sakr M, Wardrope J, et al. Thrombolysis in acute myocardial infarction: the safety and efficiency of treatment in the accident and emergency department. J Accid Emerg Med 1999;16:325-30.

11 Birkhead JS. Trends in the provision of thrombolytic treatment between 1993 and 1997. Myocardial Infarction Audit Group. Heart 1999;82:438-42.

12 Department of Health. NHS Direct. London: Department of Health, 2003

13 Pitt K. Prehospital selection of patients for thrombolysis by paramedics. Emerg Med J 2002;19:260-3.

14 Weaver WD, Cerqueira M, Hallstrom AP, et al. Prehospital-initiated vs hospital-initiated thrombolytic therapy: the Myocardial Infarction Triage and Intervention Trial. JAMA 1993;270:1211-16.

15 The European Myocardial Infarction Project Group. Prehospital thrombolytic therapy in patients with suspected acute myocardial infarction. N Engl J Med 1993;329:383-9

16 GREAT group. Feasibility, safety, and efficacy of domicillary thrombolysis by general practitioners: Grampian Region Early Anistreplase Trial. BMJ 1992;305:548-53

17 Boland A, Dundar Y, Bagust A, et al. Early thrombolysis for the treatment of acute myocardial infarction: a rapid and systematic review. Health Technol Assess (in press). (Accessed via the National Institute for Clinical Excellence web site http://www.nice.org.uk/). 